\title{
Naturaleza y especificidad de las Investigaciones en la Facultad de Trabajo Social en la Universidad Nacional del Centro del Perú
}

\author{
Kiki kayninwan achkap lulayćhu Pirup ćhawpinćhu \\ llapansuyup hatun Yaćhaywasichu
}

\section{Okantageta ontanepage ora ogomentotsipongo Universidad Nacional del Centro del Pero}

\author{
Recibido: 20 marzo 2020 Corregido: 13 mayo 2020 Aprobado: 15 junio 2020 \\ Ricardo Walter Soto Sulca \\ Nacionalidad: Peruana / Universidad Nacional del Centro del Perú \\ Correo: rsoto@uncp.edu.pe. /ORCID: https://orcid.org/oooo-0002-9936-0609
}

Julissa Karent Muñoz Rojas

Nacionalidad: Peruana / Universidad Nacional del Centro del Perú

Correo:jmunioz@uncp.edu.pe /ORCID: https://orcid.org/oooo-ooo3-3320-5064

\section{Resumen}

Nuestra intención en esta investigación es conocer la producción intelectual de los docentes de la Facultad de Trabajo Social entre los años 1978-1982, en este periodo los docentes han aportado en la discusión sobre la naturaleza de Trabajo Social, el proceso metodológico y el desarrollo de las prácticas profesionales que nos permite proponer en primera línea una reflexión sobre la producción académica de los docentes en un proceso de globalización siendo necesario tener una nueva lectura de nuestra carrera. Precisamente, el objetivo de esta investigación consiste en situar los artículos y los debates en torno a la naturaleza, metodología, prácticas-supervisión en Trabajo Social que están publicados en la revista UNITS de la UNCP entre los años 1978-1982. En este sentido, los trabajos de investigación sobre la naturaleza de la carrera profesional desarrollado por los docentes de la Facultad de Trabajo Social tienen vigencia y pueden ser debatidos y a veces crear expectativas dicho conocimiento es indispensable por la contribución en la formación académica de los estudiantes, egresados y docentes en donde, actualmente se va perdiendo el pensamiento crítico. Después de la década de los noventa se han dado cambios importantes en el mundo global donde los paradigmas se destruyen, las utopías desaparecen en las ciencias sociales comienzan a mirar del conocimiento teórico al pragmatismo instrumental. El Trabajo Social no es ajeno a todo el discurso del pragmatismo instrumental que se vienen implementando en todas las ciencias sociales, dejando de lado la búsqueda de una teoría crítica que puede servir como principio o filosofía y oriente nuestra intervención.

\section{Palabras clave:}

Naturaleza, metodología, supervisión, prácticas profesionales, trabajo social.

\section{Lisichiku limaykuna:}

Kaynin, lulana, likapaana, lulakuna yaćhapaku, achkap lulay.

\section{Nibarintsipage katingatsaro: okantagitetageta okogane netsagantsiage. Antagantsipage okantakagantageti kara paretagetari}

\section{Datos de los autores}

Ricardo Walter Soto Sulca es docente e investigador de la Facultad de Trabajo Social, en temas: Trabajo Social, Sociología de Infancia, Estudios Culturales, Política. Magister en Sociología por la Pontificia Universidad Católica del Perú.

Julissa Karent Muñoz Rojas, es docente e investigador de la Facultad de Trabajo, líneas de investigación en trabajo social; desarrollo humano y políticas sociales. Magister en Políticas Sociales con mención en Promoción de la Infancia por la Universidad Nacional del Centro del Perú. 


\section{Nature and Specificity of Research at the Faculty of Social Work at the National University of Central Peru}

\begin{abstract}
Our intention in this investigation is to know the intellectual production of the teachers of the Faculty of Social Work between the years 1978-1972, in this period the teachers have contributed in the discussion about the nature of Social Work, the methodological process and the development of the professional practices that allow us to propose in the first line a reflection on the academic production of teachers in a process of globalization, being necessary to have a new reading of our career. Precisely, the objective of this research is to place articles and debates around the nature, methodology, practices-supervision in Social Work that are published in the UNITS magazine between the years 1978-1982. In this sense, the investigation work on the nature of the professional career developed by the faculty of the Social Work Faculty are valid and can be debated and sometimes create expectations such knowledge is indispensable for the contribution in the academic formation of the students, graduates and teachers, is currently losing critical thinking. After the nineties there have been important changes in the global world where paradigms are destroyed, utopias disappear in the social sciences, they begin to look from theoretical knowledge to instrumental pragmatism. Social Work is not alien to all the discourse of instrumental pragmatism that is being implemented in all social sciences, leaving aside the search for a critical theory that can serve as a principle or philosophy and guide our intervention.
\end{abstract}

\section{Keywords}

Nature, Methodology, Supervision, Professional Practices, Social Work

\section{Natureza e especificidade das pesquisas na Serviço Social na Universidad Nacional del Centro del Perú-UNCP}

\section{Resumo}

Nossa intenção nesta investigação é conhecer a produção intelectual dos professores da Faculdade de Serviço Social entre os anos de 1978-1982, nesse período os professores têm contribuído na discussão sobre a natureza do serviço social, o processo metodológico e o desenvolvimento das práticas profissionais que nos permitem propor em primeira linha uma reflexão sobre a produção acadêmica dos professores em um processo de globalização, sendo necessário ter uma nova leitura de nossa carreira. Precisamente, o objetivo desta pesquisa é colocar artigos e debates em torno da natureza, metodologia, práticas-supervisão no serviço social que são publicados na revista UNITS da UNCP entre os anos de 1978-1982. Nesse sentido, os trabalhos de investigação sobre a natureza da carreira profissional desenvolvida pelo corpo docente da Faculdade de Serviço Social tem vigência e pode ser debatido e, por vezes, criar expectativas de que tal conhecimento é indispensável para a contribuição na formação acadêmica dos alunos, graduados e professores, onde atualmente está se perdendo o pensamento crítico. Após os anos noventa, houve mudanças importantes no mundo global, onde paradigmas são destruídos, utopias desaparecem nas ciências sociais, começam a olhar do conhecimento teórico ao pragmatismo instrumental. O serviço social não é alheio a todo o discurso do pragmatismo instrumental que está sendo implementado em todas as ciências sociais, deixando de lado a busca de uma teoria crítica que possa servir de princípio ou filosofia e orientar nossa intervenção.
Palavras-chave:

Natureza, metodologia, supervisão, práticas profissionais, serviço social 


\section{Introducción}

Al Trabajo Social se le asigno históricamente la función de atender, de neutralizar el desorden social provocado por la desigualdad e injusticia social; con la finalidad de lograr la disminución del daño, incluido el daño afectivo, emocional que acarrean circunstancias de la vida al límite de la sobrevivencia humana en contextos de desigualdad e injusticia social (Cusianovich, 2016).

Desde mucho tiempo se viene afirmando al Trabajo Social como una disciplina de las Ciencias Sociales no cuenta con teoría propia, metodología, sino que utiliza las diversas teorías y metodologías de la sociología, antropología, psicología, administración entre otros. Con la presente investigación podemos conjeturar que los docentes han tenido una visión dogmática y mecánica de la realidad social y principalmente de la carrera porque para su análisis utilizaban el marxismo como la única teoría de las ciencias sociales que creían científica, viéndose reflejado en que los docentes de esa época no tuvieron una mirada amplia y compleja de la carrera.

Hasta hace algunos años, parecía que la discusión en torno a los temas como: la naturaleza, la metodología y la práctica del Trabajo Social, ya no era necesaria, ante el ascenso y afianzamiento del nuevo orden mundial, los cambios abruptos sucedidos en el ámbito local, global; sobre los embates que sufren las ciencias sociales en general, y el materialismo histórico y dialéctico, en particular (Montaño 2000). Sin embargo, en la actualidad ha vuelto a ser importante tener claro la naturaleza de nuestra carrera. Eso trajo consigo una serie de tendencias que llevaron a afianzar el positivismo y su variante funcionalista, y se puso en entredicho la validez de una serie de teorías y metodologías que surgieron de la reconceptualización del Trabajo Social.

Al interior de la profesión, el debate sobre la naturaleza y especificidad cobra vigencia en el momento actual, y evidencia el repliegue estratégico de los seguidores de la corriente científica del materialismo histórico y dialéctico, al repensar el Trabajo Social en el marco de las ciencias sociales, compartiendo o no su anunciada crisis, pero de todos modos revitalizando la discusión de viejas teorías con nuevos enfoques y condicionalidades. Surgen nuevos planteamientos sobre si es o no una disciplina, sin cambiar la tendencia de que es una disciplina en camino de construcción y también se da la discusión sobre cuál es su ámbito de acción, la generalidad de lo social o bien lo singular de la problemática social dentro de las oleadas neoliberales (Montaño, 1998).

La presente investigación pretende analizar la producción intelectual de los docentes a partir de los artículos de la revista UNITS que la Facultad de Trabajo Social publicaba entre los años 1978-1982, etapa primordial donde se encuentran los debates sobre la Naturaleza de nuestra profesión, las propuestas metodológicas, la supervisión y las experiencias de las prácticas profesionales. Pretende aportar una primera reflexión sobre los diversos enfoques sobre la naturaleza de Trabajo Social, proceso metodológico, supervisión y desarrollo de las prácticas del Trabajo Social que han desarrollado los docentes de la Facultad de Trabajo Social de la UNCP entre los años 1978-1982.

Las ideas centrales expuestas en la presente investigación indudablemente podrán ampliarse o cuestionarse por los lectores, a través de la discusión epistemológica, de la investigación y sistematización, con lo cual se obtendrá la mejor contribución.

Teniendo como interrogante general ¿Cuáles son los diversos enfoques de la naturaleza, metodología y las prácticas del Trabajo Social que han desarrollado los docentes de la Facul- 
tad de Trabajo Social de la UNCP entre los años 1978-1982?, y teniendo como objetivo general: analizar los diversos enfoques de la naturaleza, proceso metodológico y las prácticas del Trabajo Social que han desarrollado los docentes de la Facultad de Trabajo Social de la UNCP entre los años 1978-1982.

Podemos llegar a la conclusión de nuestra investigación que las dos tendencias tienen como trasfondo una concepción marxista, para entender la naturaleza de la carrera es necesario demarcar la diferencia entre los términos: profesión y profesional; pues para la presente investigación los resultados demuestran que no se ha diferenciado la profesión con el profesional; pues, la profesión te da un conjunto de conocimientos teóricos diversos, metodologías de intervención clásicas y contemporáneas, contar con un sistema de prácticas, etc. En cambio, el término profesional es definido como el actor quien puede desarrollar cualquier teoría o metodología según el sector que trabaja o desarrolle sus prácticas, este actor puede tener una opción política partidaria, que lo relaciona entre la política y su quehacer profesional que es de entera responsabilidad del profesional, no tiene nada que ver la carrera profesional.

Asimismo quisiéramos señalar dos elementos importantes, la primera es comprender los diversos enfoques sobre la naturaleza, metodología y de las prácticas del Trabajo Social que han desarrollado los docentes de la Facultad de Trabajo Social de la UNCP entre los años 1978-1982, y la segunda, está basada en la reflexión seria de la producción académica de los docentes que tienen del Trabajo Social en un proceso de globalización donde los paradigmas teóricos son cada vez más flexibles y cambiantes esto nos hace necesario para tener una nueva lectura de nuestra carrera.

\section{Métodos y materiales}

La investigación es cualitativa basado en la revisión y análisis documentaria de fuente primarias y secundarias. Comenzando con las fuentes internas, hemos privilegiado los testimonios de los docentes que estuvieron entre los años 1978-1982 en actividad en la facultad de trabajo social; entrevistamos a total de 4 docentes. La segunda fuente principal, es la revisión documentaria, el análisis de la revista UNITS se convirtió en el soporte fundamental para estructurar la investigación. Se utilizó algunas categorías básicas para entender la producción intelectual de los docentes, como el contexto político de la época, la naturaleza de la profesión de Trabajo Social, la metodología de Trabajo Social, las supervisiones de Trabajo Social, las experiencias de las prácticas en diversos sectores de intervención profesional. Por esta razón a lo largo de la investigación las referencias a la revista son casi permanentes. Los cuatro números componen la colección de la revista UNITS constituyen sin duda la mejor fuente para el estudio del tiempo de la producción científica de los docentes. Es muy encomiable el trabajo que desarrollaron los docentes en los cinco años que duro la revista con artículos, ensayos, etc.

\section{Discusión de resultados}

Nos han parecido sumamente importante los hallazgos que se han obtenido de una investigación casi documental sobre la producción intelectual de los docentes de Trabajo Social en la revista de investigación UNITS donde sus artículos y debates giraban en los temas de la naturaleza del Trabajo social y el proceso metodológico de la carrera. Pasamos a desarrollar la discusión de nuestros resultados con nuestros aparatos teóricos que se han utilizado a lo largo de nuestra investigación. 


\section{Naturaleza de la profesión}

Como podemos encontrar en los artículos de los cuatro números de la revista UNITS el debate entre los docentes de la facultad sobre el carácter o la naturaleza de Trabajo Social tiene un asidero teórico que es el movimiento de la reconceptualización como señala (Micheline, El Servicio Social y el papel de las ideas en el desarrollo social, 1978):

En el servicio social existen básicamente dos tendencias: a.- La tendencia, cuyo objetivo es eminentemente político al servicio de las masas explotadas y que plantea la contribución profesional a la transformación de las estructuras socio- económicas vigentes. b.- La tendencia en la que consiste en impulsar la reconceptualización en servicio social pero como una reivindicación de los profesionales ideología y cultura opresora "... Donde toma vigencia la lucha por el avance científico de la profesión, sea esto en el plano de la teoría, concepción del mismo desarrollo de su método, por lo mismo es una lucha democrática. Sostienen que las reivindicaciones deben ser impulsadas desde el seno del pueblo y que no pueden ser objetivos de orden profesional".

\section{Como nos dice (Tanaka, 2016):}

Para que ocurra un debate alguien debe considerar muy importante desmontar la argumentación de algún otro o proponer explicaciones o ideas alternativas, motivado ya sea por un valor pedagógico como por lograr que se imponga una posición particular o de grupo sobre algún asunto considerado especialmente importante. Para que un debate se produzca debe existir además de un contexto plural y diversos de posiciones y cierto grado de polarización. Así puede haber muchos temas de controversia, sin embargo, no todos generan polémicas: ellas nos sugieren algunos ejes de tensión o conflicto que atravesaron a la comunidad de ciencias sociales en los últimos años.

Podemos conjeturar que las dos tendencias tienen como trasfondo una concepción marxista para poder entender la naturaleza de la carrera, porque no diferenciaban la profesión con el profesional, la profesión te da un conjunto de conocimientos teóricos diversos, metodologías de intervención clásicas y contemporáneas, cuentas con un sistema de prácticas, etc. En cambio, el profesional como un actor puede desarrollar cualquier teoría o metodología según el sector que trabaja o desarrolla sus prácticas, este actor puede tener una opción política partidaria, que lo relaciona entre la política y su quehacer profesional el cual es de entera responsabilidad del profesional, no tiene que ver la carrera profesional.

Como se describe los docentes en su mayoría tenían una formación dogmática de la teoría marxista porque para ellos tenían una concepción economicista de la realidad social como ellos lo explican (Estrada,G. Maravi,L, 1979):

Con estas afirmaciones llegamos a la conclusión implícita que mientras no cambie la base económica de nuestra sociedad, la profesión no puede contribuir a la transformación del orden vigente por su carácter de clase y por qué no debe confundirse los objetivos profesionales con los del partido político del proletariado. Es así que sostienen: "Lo que plantea en esencia esta tendencia es un cambio de carácter mismo de clases de la profesión, sin tener en cuenta lo que la teoría marxista plantea sobre la relación existente entre la base económica y la superestructura en sus niveles políticos, jurídico e ideológico".

El mismo (Tanaka, 2016) explica:

Los intelectuales tienen una mirada mecánica de la realidad Como veremos, los análisis 
aparecen asociados a miradas voluntaristas e ideologizadas que no eran capaces de considerar toda la evidencia disponible y que además miraban la realidad desde lentes conceptuales que sesgaban las lecturas de los procesos en curso; correlativamente, las miradas más profundas están asociadas a un equilibrio entre un compromiso político y un conocimiento científico de la realidad social y política y a miradas más informadas en lo teórico y conceptual, más en dialogo con perspectivas comparadas, más articuladas a la academia regional y global.

Igualmente podemos conjeturar que esta mirada dogmática de la realidad les hizo perder el norte de la profesión porque el desarrollo del Trabajo Social no tiene una relación con la economía de una sociedad, sino por el contrario el conocimiento influye en la economía, la política, lo social y ambiental estos aspectos señalados van de la mano entre ellos. Nosotros creemos que las teorías tienen un alcance limitado, por tanto, el profesional para su intervención puede utilizar diversas teorías no solo una teoría totalizante al cual Ilamaban "científica".

\section{Metodología de trabajo social}

En cuanto a la metodología de la carrera profesional también los docentes de la facultad tuvieron dos miradas: la primera basada en la metodología tradicional de caso, grupo y comunidad y la segunda la metodología contemporánea como el básico, integrado y la de investigación y acción. Pero la interior de estas dos miradas se encontraba implícitamente o explícitamente el concepto de asistencialismo. Como lo señalan los docentes (Contreras, 1981):

El Servicio Social Tradicional en relación al campesino se ha limitado sólo a buscar la orientación de los problemas que sufre la población rural; mediante el asistencialismo y la utilización de una teoría funcionalista sin realizar una investigación e interpretación de los problemas, tal así, que para su alivio utilizaban los métodos: Caso, Grupo y Comunidad.

La otra mirada de un asistencialismo científico está relacionada con la metodología como lo menciona una de las docentes (Vasquez, 1978):

Este asistencialismo dentro del desarrollo científico de la profesión, también se ha modificado, es decir, ya no será un asistencialismo filantrópico, sino, que se fundará en la atención de los problemas que aquejan al hombre en función de su objetivo establecido, real y concreto -programas de bienestar social-. Es decir, ya no es accionar profesionalmente para dar dádivas a los necesitados, sino actuar para orientar a los necesitados en función de los Derechos qué estipula la ley, sin que esto quiera decir, que de esta manera solucionamos los problemas.

Como nos señala (Ibañez, 1994):

El Trabajo Social, es más dedicado a hacer que a pensar cómo lo hace, distintos momentos o procesos fueron confundidos como métodos en sí mismos. Y frente a cada unidad de abordaje se fueron creando métodos. Así fueron surgiendo los de caso social individual, grupo y comunidad, mientras se consideraron métodos auxiliares la investigación, supervisión y administración, sin advertir que los primeros no eran métodos y los segundos son instancias, etapas o momentos del método.

Podemos conjeturar, que hasta la actualidad no se ha podido deslindar si es pertinente o no, la metodología tradicional de caso social, grupo y comunidad o las otras propuestas metodológicas de intervención que aparecieron en el trascurso de la historia de Trabajo Social. 
En estos momentos los profesionales de la carrera utilizan diversas metodologías según su campo de intervención no por ello su labor profesional deja de ser de calidad, por otra parte, otras profesiones también utilizan los métodos tradicionales de caso, grupo y comunidad, sin embargo, tienen otros objetivos y metas. Por lo que podemos afirmar en esta coyuntura no existe como propiedad exclusiva una sola metodología en la carrera y en las otras carreras profesionales.

\section{Experiencia de prácticas profesionales}

En la revista UNITS en sus cuatro números dan cuenta de las experiencias de prácticas profesionales que desarrollan los docentes y estudiantes, los primeros como supervisores de prácticas y los alumnos como ejecutores de las prácticas en las diversas áreas de intervención.

Podemos constatar que el debate de la naturaleza y la metodología de trabajo social que se daba en las aulas universitarias, buscaban poner en práctica en los diversos niveles de prácticas de los estudiantes, es decir los docentes y alumnos desarrollaban la propuesta metodológica de investigación-acción-sistematización con un enfoque teórico marxista de contribuir a la trasformación de la sociedad, otros docentes y estudiantes persistían en la metodología de caso, grupo y comunidad con un enfoque bienestar social. Una de los docentes manifiesta (Micheline, Tareas del Trabajo Social comprometido, 1982):

El trabajo social es replanteado como una profesión comprometida con el desarrollo de la sociedad y de orientación de trabajo hacia las clases populares, es necesario que dicha acción este sustentada por una formación sólida que le permita una mayor eficacia cualitativa y no cuantitativa, que la práctica no sea un mero activismo sino una práctica científica sustentada por una teoría basada en la filosofía marxista, por lo que la formación profesional está dirigida en dos niveles: La teoría y práctica. “La teoría depende de la práctica, que la base de la teoría es la práctica y que no teoría a su vez, sirve a la práctica.

Como también lo señala la docente (Manrique, 1981).

Necesidad de una metodología de acción que no sea una simple readecuación de métodos y técnicas tradicionales de caso, grupo y comunidad o la elaboración de un método básico qué parte de los mismos supuestos teóricos tradicionales, si no que sea un producto de un análisis y conocimiento de la realidad de tal manera, que ya pueda constituirse en una metodología para la acción y que pueda permitir la construcción de una teoría de trabajo social.

Como se puede constatar en el debate existía una fuerte ideologización en los docentes y alumnos porque muy mecánicamente llevaban sus propuestas teóricas y metodológicas al desarrollo de sus prácticas sin tener en cuenta la dinámica social de las instituciones y pobladores porque ellos tenían otras finalidades, en muchos casos los alumnos por cumplir sus prácticas forzaban a la población para poder calzar con los interés de la carrera y no de los usuarios, esto nos ha llevado hasta la actualidad no poder articular entre la academia y la sociedad.

\section{Conclusiones}

Las dos tendencias tienen como trasfondo una concepción marxista para entender la naturaleza de la carrera, no han diferenciado la profesión con el profesional, la profesión te da un conjunto de conocimientos teóricos diversos, metodologías de intervención clásicas y con- 
temporáneas, cuentas con un sistema de prácticas, etc. En cambio, el profesional como un actor puede desarrollar cualquier teoría o metodología según el sector que trabaja o desarrolla sus prácticas, este actor puede tener una opción política partidaria, que lo relaciona entre la política y su quehacer profesional es de entera responsabilidad del profesional, no tiene que ver la carrera profesional.

Hasta la actualidad no se ha podido deslindar si es pertinente o no, la metodología tradicional de caso social, grupo y comunidad o las otras propuestas metodológicas de intervención que aparecieron en el trascurso de la historia de Trabajo Social. En estos momentos los profesionales de la carrera utilizan diversas metodologías según su campo de intervención no por ello su labor profesional deja de ser de calidad, por otra parte, otras profesiones también utilizan los métodos tradicionales de caso, grupo y comunidad, pero tienen otros objetivos y metas por lo que podemos afirmar en esta coyuntura no existe como propiedad exclusiva una sola metodología en la carrera ni en las otras carreras profesionales.

Las prácticas son consideradas de corte transcendental para la formación del estudiante, desde el inicio de la carrera, su particularidad con las otras carreras de las ciencias sociales es la práctica profesional donde se aplica todas los saberes y conocimientos previos para la intervención profesional, entre los años que 1978-1982, las prácticas en Trabajo Social se desarroIlaron teniendo en cuenta la naturaleza y metodología de trabajo social en diversos campos laborales privados o públicos, concluyendo que fue una experiencia muy ideologizada fuera de los intereses de la población beneficiaria.

\section{Referencias bibliográficas}

Contreras, N. (1981). La teoría funcionalista y su aplicación en practicas en Trabajo Social en comunidades. UNITS 3, 26-31.

Cusianovich, A. (2016). Pedagogia de la ternura y Trabajo Social. Praxis Social, 107-125.

Estrada,G. Maravi,L. (1979). Caracter de Clase del Servicio Social. UNITS 2, 15-22.

Ibañez, T. (1994). Psicologia Social Construccionista. Guadalajara - Mexico: Universidad de Guadalajara.

Manrique, A. (1981). A proposito de la pretendida concientización del pueblo para su liberación. UNITS - 3, 5-13.

Micheline, E. (1978). El Servicio Social y el papel de las ideas en el desarrollo social. UNITS-1, 56-72.

Micheline, E. (1982). Tareas del Trabajo Social comprometido. UNITS - 4, 34-61.

Montaño, C. (1998). Naturaleza del Servicio Social. San Pablo-Brasil: Cortez.

Tanaka, M. (2016). Una evaluación post-factum de los grandes debates políticos en la Facultad de Ciencias Sociales de la PUCP. En F. Alan, El Perú visto desde las Ciencias Sociales. Lima: Fondo editorial PUCP.

Vasquez, M. (1978). Practicas en el sector rural-UNCP. UNITS - 1, 23-36. 